\title{
Analyse eines Dokumentarfilms oder eines Ausschnitts aus einer Informationssendung im GW-Unterricht
}

\author{
p.hofmann@tsn.at, BG/BORG St. Johann in Tirol; Institut für fachdidaktische und bildungswissenschaftliche Forschung \\ und Entwicklung, Pädagogische Hochschule Tirol
}

eingereicht am: 01.05.2020, akzeptiert am: 20.05.2020

\begin{abstract}
Dieser Servicebeitrag bietet eine Anleitung zur kritischen Analyse von Dokumentarfilmen und Videoclips im Unterricht in der Form eines Arbeitsblattes, bestehend aus sieben Elementen: Grundinformationen, formale Kriterien, Inhaltszusammenfassung, Inhaltsbeschreibungen der einzelnen Sequenzen, kritische Anmerkungen und Interpretationen, eigene Meinung und kritischer Vergleich mit anderen Videos.
\end{abstract}

Keywords: Medien, Dokumentarfilm, kritische Filmanalyse, GW-Unterricht

\section{Analysis of a documentary film or a sequence of a geography-based series in geography and economics lessons}

This contribution provides a guide for a critical analysis of geography-based documentaries and video clips in class. The worksheet contains: basic information, formal criteria, a short summary, content descriptions for each sequence, critical remarks and interpretations, the students' own opinion and a critical comparison with other videos.

Keywords: media, critical analysis of documentary films and video clips, education in geography and economics

\section{Einleitung}

Es ist eine erfreuliche Tatsache, dass sich in den letzten Jahren im Zuge des kompetenzorientierten Unterrichts auch in Geographie und Wirtschaftskunde eine Verstärkung des Einsatzes und der Vermittlung von fachrelevanten Arbeitsmethoden durchgesetzt hat. Diese Vermittlung von Arbeitsmethoden fand zunehmend seit rund 15 Jahren auch in vielen Schulbüchern, mit eigenen, meist farblich hervorgehobene Methodenseiten oder -abschnitten, Eingang und reicht vom Kartenlesen, der Satellitenbildauswertung, der Erstellung und Auswertung von Diagrammen und Texten bis hin zur Interview- und Fragebogengestaltung und Bildauswertung (ohne hier alle Methoden aufzuzählen). Ein Medium, das auch in GW sehr gerne verwendet wird, sind Dokumentarfilme und Videoclips in einem schier unübersichtlichen Angebot (von bereits vorsortierten und geordneten Angeboten von Education-Sites, wie die gerade kürzlich, situationsbedingt neu hinzugekommene „edutube“, bis hin zur Flut von Clips in „YouTube“).
Es gibt auch einige Dokumentarfilme, die bereits Arbeitsblätter für den Schulgebrauch auf eigenen Webseiten anbieten, passend zum Film. Die Zahl letzterer ist aber überschaubar und manche sind nicht mehr ganz zeitgemäß.

Damit Filme und Videoclips - wie früher die Fotos in den Schulbüchern - nicht nur den Charakter einer zusätzlichen visuellen Übersetzung bereits präsentierter Unterrichtsinhalte haben oder gar nur als Lückenfüller eingesetzt werden, braucht es auch hier eine kurze Methodenbeschreibung bzw. ein universell einsetzbares Arbeitsblatt, mit dem Schüler/innen Dokumentationen oder Clips aus Informationssendungen kompetenzorientiert bearbeiten können. Leider ist dem Autor eine derartige Methodenbeschreibung bzw. ein solches Arbeitsblatt in keinem aktuellen GW-Buch untergekommen, oder er hat beides leider übersehen. Als Verfasser eines inzwischen nicht mehr aktuellen und deshalb aktiv aus der Schulbuchliste genommenen Schulbuches, hat es der Autor seinerzeit leider selber auch verabsäumt, eine derartige Methode genauer zu beschreiben. 
Kolleginnen und Kollegen, welche Vorlagen zu kritischen Filmanalysen jenseits von reinen Nacherzählungen und schriftlichen Zusammenfassungen schon selbst zusammengestellt haben und diese bereits jetzt professionell einsetzen, werden hier nicht viel Neues finden. Das Angebot ist vor allem als Service für jene gedacht, die dies bisher im GW-Unterricht noch nicht so sehr ins Auge gefasst haben.

Im Folgenden wird kurz ein möglicher Arbeitsauftrag bzw. ein Arbeitsblatt vorgestellt, mit dem im Prinzip die meisten in GW verwendeten Videoclips bearbeitet werden können. Es ist allerdings auch in anderen Fächern (z. B. Biologie und Umweltkunde oder Geschichte und politische Bildung) für die Analyse von filmischen Dokumentationen einsetzbar. Dies ist sowohl im normalen Präsenzunterricht als auch als digitaler Arbeitsauftrag möglich, der zusammen mit dem Video auf eine Lernplattform gestellt werden kann.

\section{Arbeitsblatt}

Das Arbeitsblatt gliedert sich in folgende sechs bzw. sieben Abschnitte:

- Grundinformationen,

- formale Kriterien,

- eine kurze Inhaltszusammenfassung des gesamten Films,

- inhaltliche Beschreibung unterteilt in Sequenzen,

- kritische Anmerkungen und Interpretation dazu,

- eigene Meinung zum Film

- und einem letzten Abschnitt zum kritischen Vergleich von Filmen, der nur dann relevant wird, wenn zwei oder mehrere Beiträge miteinander verglichen werden sollen.

Die vorgestellte Version des Arbeitsblattes eignet sich für alle Klassen der Sekundarstufe II. Für Klassen der Sekundarstufe I muss das Arbeitsblatt an die Leistungsfähigkeit der Schülerinnen und Schüler von der Lehrperson sicherlich angepasst werden.

Bei den ersten beiden Abschnitten Grundinformationen und Formale Kriterien können möglicherweise viele Informationen gar nicht erhoben werden und sind dann einfach mit der Bemerkung „Keine Angabe gefunden" zu versehen. Oder man findet nur Teilinformationen (z. B. den Namen des Regisseurs oder Moderators, aber keine Informationen über ihn). Die fett hervorgehobenen Kategorien können aber auf jeden Fall bearbeitet werden.

\section{Tipps zur Durchführung}

Es ist auch anzuraten, nicht den gesamten Film (wenn es sich um eine längere Dokumentation handeln sollte) von einem Schüler bzw. einer Schülerin durchführen zu lassen, sondern die genauen Sequenzanalysen auf die Schüler/innen aufzuteilen, auch wenn jeder die gesamte Dokumentation einmal im Ganzen gesehen haben sollte. Hier kann man mit verschiedenen Varianten arbeiten: Entweder suchen sich die Schüler/ innen selbst zwei oder drei Sequenzen aus, oder die Lehrperson unterteilt im Vorfeld den Film in Einzelsequenzen und ordnet den Schülern/Schülerinnen dann bestimmte Sequenzen zur genaueren Analyse zu. Bei kürzeren Videoclips (z. B. aus Nachrichtensendungen), die nur wenige Minuten dauern, ist dies nicht notwendig.

Es ist anzuraten, für die Umsetzung eine Datei zu haben, die ausgedruckt und handschriftlich bearbeitet werden kann, indem die Schüler/innen parallel zum Schauen des Videos dieses immer wieder stoppen und handschriftliche Anmerkungen machen können. Das Endresultat sollte dann aber Präsentationsqualität haben, entweder in Form eines digitalen Dokuments (z. B. Word-Dokument) oder auch in Form einer Präsentation (z. B. Powerpoint).

Abschließend ist noch zu bemerken, dass eine derartige kritisch-orientierte Filmanalyse nur dann möglich ist, wenn das Videomaterial jeder Schülerin / jedem Schüler einzeln zur Verfügung gestellt werden kann bzw. zugänglich ist. Das ist vor allem dann möglich, wenn das Material auf kostenlosen Websites angeboten wird. Jede und jeder muss individuell das Video vor- und zurückspielen können. Hiermit fällt eine derartige Analyse für einen von den Lernenden gemeinsam in der Klasse gesehenen Filmbeitrag aus, außer das Filmmaterial kann nachträglich für alle zugänglich gemacht werden.

Eine mögliche Abwandlung bzw. Erweiterung des Arbeitsauftrages besteht vor allem darin, dass man zwei oder mehrere filmische Beiträge zum gleichen Thema (z. B. aus zwei verschiedenen Nachrichtensendungen) miteinander vergleichen lässt. Hier würde dann noch die siebte Kategorie im Arbeitsblatt hinzukommen: Kritischer Vergleich der Inhalte. 


\title{
ARBEITSBLATT ZUR FILMANALYSE
}

\section{Grundinformationen}

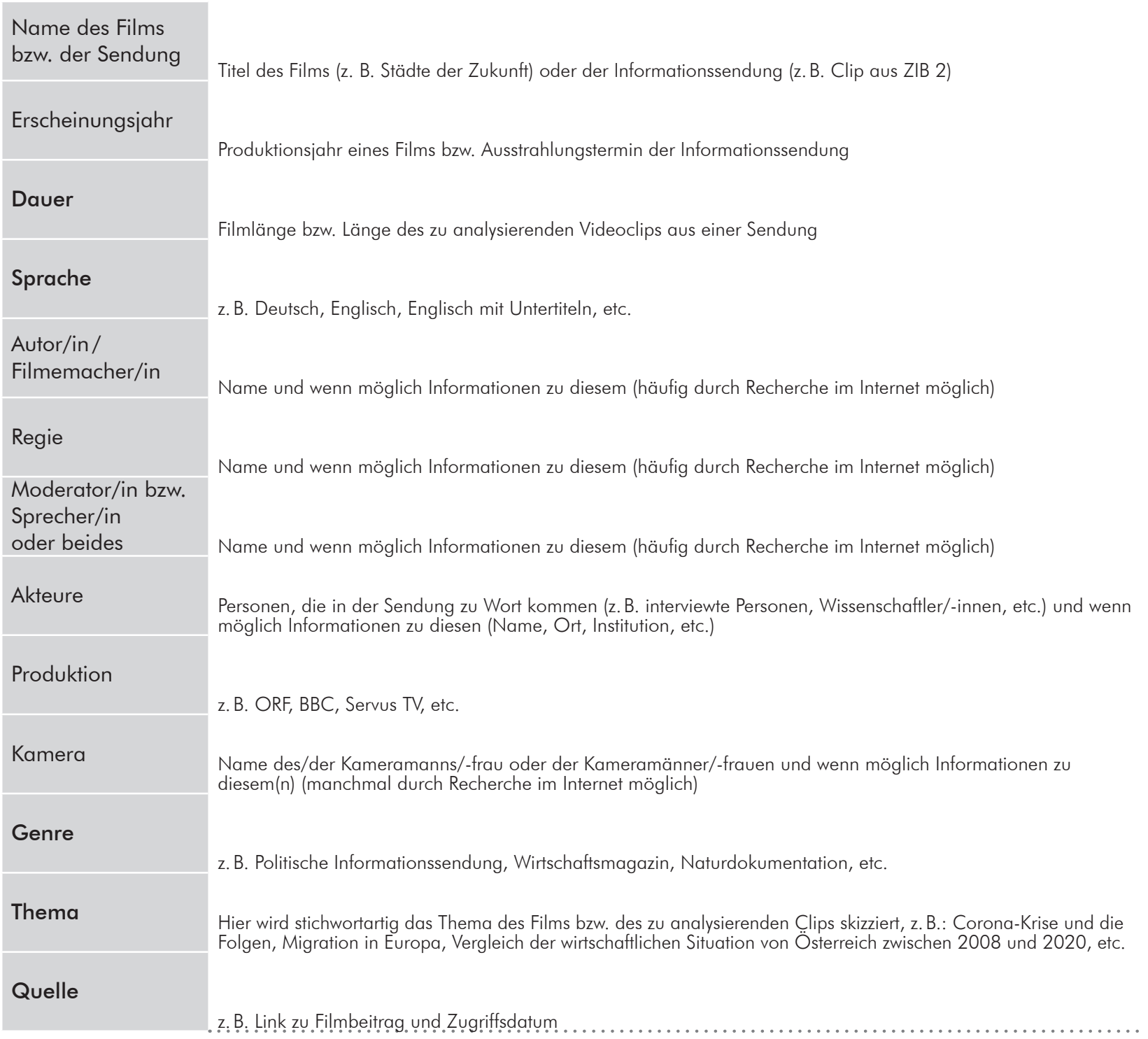

\section{Formale Kriterien}

\author{
Bildqualität \\ (Auflösung)

\section{Farben} \\ Hier wird die Qualität angegeben, z. B. 1280×720, 1024×576 Pixeln, Full HD, etc.

Kameratechnik \\ Angabe ob SW oder zur Farbqualität (individuelle Einschätzung wie z. B. blass, sehr intensiv, etc.)

\section{Musik und Ton} \\ Beschreibung der Kameraführung bzw. ob Spezialeffekte verwendet werden (z. B. Luftbildaufnahmen, etc.)

\section{Filmschnitt} \\ Angaben zu Verwendung von Musikuntermalung, Tonqualität \\ Schauplätze \\ Angaben zu Auswahl und Strukturierung des Bild- und Tonmaterials (z. B. Wechsel zwischen Aufnahmen eines \\ Moderators / einer Moderatorin und Aufnahmesequenzen einer Landschaft)




\section{Kurze Zusammenfassung des Filminhalts}

Hier sollte eine relativ kurze inhaltliche Zusammenfassung des Videoclips oder des Dokumentarfilms stehen, die einen raschen Überblick über den Inhalt gibł z. B.

- 5 min Beitrag aus ZIB 2 zur Arbeitsmarktsituation in Österreich im April 2020 oder

- 15 min Ausschnitt aus 50 min Dokumentarfilm über den Nationalpark Hohe Tauern mit Schwerpunktthemen Naturschutz, Artenvielfalt und Bedeutung für den Tourismus

- 30 min Dokumentation über die Sahelzone, ihre Problematik im Zuge des Klimawandels und die Folgen für die dortige Bevölkerung.

$\therefore$ usw.

Die folgenden Abschnitte dienen einer genaueren Analyse des Videomaterials, indem es in Sequenzen unterteilt wird. Eine solche Sequenz besteht aus aufeinanderfolgenden Filmeinstellungen, die grafisch, räumlich, zeitlich, thematisch, szenisch oder unter Aspekten zusammengehören und durch einen in sich abgeschlossenen filmischen Abschnitt im Lauf der Dokumentation darstellen.

\section{Inhaltliche Beschreibung (unterteilt in Sequenzen)}

\section{Sequenz 1 \\ Zeit: \\ Position im Film:}

Filmischer Inhalt
Eine Bezeichnung dafür wählt der Schüler/die Schülerin selbst.

z. B. zwischen $12 \mathrm{~min} 40 \mathrm{sec}$ und $17 \mathrm{~min} 24 \mathrm{sec}$

Hier wird angegeben, wo sich die Sequenz befindet (z. B. Einleitung, Hauptteil, Zusammenfassung) Kommentar ("Was hört man dazu?")
Schüler/Schülerin schreibt hier eine Beschreibung des gesehenen Inhalts (Der Videoclip sollte dabei am besten ohne Ton abgespielt werden, damit der Kommentar niçht ablenkt)
: Gleiche Sequenz wird mit Ton abgespielt und eine kurze Zusammen: fassung. des Inhalts gegeben.

\section{Sequenz 2}

Zeit:

Eine Bezeichnung dafür wählt der Schüler/die Schülerin selbst.

Zeit:

Position im Film:

Filmischer Inhalt

z. B. zwischen $12 \mathrm{~min} 40 \mathrm{sec}$ und $17 \mathrm{~min} 24 \mathrm{sec}$

Hier wird angegeben, wo sich die Sequenz befindet (z. B. Einleitung, Hauptteil, Zusammenfassung)

Schüler/Schülerin schreibt hier eine Beschreibung des gesehenen Inhalts (Der Videoclip sollte dabei am besten ohne Ton abgespielt werden, damit der Kommentar nicht ablenkt)

Kommentar ("Was hört man dazu?")
}

Sequenz 3, Sequenz 4, etc. 


\section{Kritische Stellungnahme zu den einzelnen Sequenzen}

\section{Sequenz 1}

- Die Szene wirkt sehr glaubhaft, weil ...

- Informationen werden sachlich und ohne eigene Meinung des Moderators/der Moderatorin präsentiert.

- Bildinformation und Kommentar passen nicht zusammen.

- Sehr einseitige Berichterstattung, weil

- Kameraschnitt konzentriert sich auf wenige Eindrücke

$\therefore$ etc.

\section{Sequenz 2}

- Die Informationen wirken seriös, weil

- Die Darstellung der Szene ist verständlich strukturiert.

- Der Sprecher/die Sprecherin spricht sehr aufgeregt.

$\therefore$ etc.

Sequenz 3, Sequenz 4, etc.

\section{Eigene Meinung zum Film}

Hier können jetzł abschließend alle Kommentare zum Film bzw. den zu bearbeitenden Ausschnitten aus dem Film zusammengefasst werden, welche die kritischen Anmerkungen von oben zusammenfasst aber auch andere Gesichtspunkte umfassen kann . (z. B. ästhetischer Eindruck et. etc.).

\section{Kritischer Vergleich der Inhalte von ...}

Bezeichnung der filmischen Beiträge, die verglichen werden

a)

b) 